\title{
Papers
}

\section{Is there a rationale for rationing chronic dialysis? A hospital based cohort study of factors affecting survival and morbidity}

Shahid M Chandna, Joerg Schulz, Christopher Lawrence, Roger N Greenwood, Ken Farrington

\begin{abstract}
Objectives To determine factors influencing survival and need for hospitalisation in patients needing dialysis, and to define the potential basis for rationing access to renal replacement therapy.

Design Hospital based cohort study of all patients starting dialysis over a 4 year recruitment period (follow up 15-63 months). Groups were defined on the basis of age, comorbidity, functional status, and whether dialysis initiation was planned or unplanned. Setting Renal unit in a district general hospital, which acts as the main renal referral centre for four other such hospitals and serves a population of about 1.15 million people.
\end{abstract}

Subjects 292 patients, mean age 61.3 years (18-92 years, SD 15.8), of whom 193 (66\%) were male, and 59 $(20 \%)$ were patients with diabetes. Dialysis initiation was planned in $163(56 \%)$ patients and unplanned in 129 (44\%).

Main outcome measures Overall survival, 1 year survival, and hospitalisation rate.

Results Factors affecting survival in the Cox's proportional hazard model were Karnofsky performance score at presentation (hazard ratio $0.979,95 \%$ confidence interval 0.972 to 0.986 ), comorbidity severity score $(1.240,1.131$ to 1.340$)$, age (1.036, 1.018 to 1.054$)$, and myeloma $(2.15,1.140$ to 4.042). The Karnofsky performance score used 3 months before presentation was significant $(0.970$, 0.956 to 0.981 ), as was unplanned presentation in this model (1.796, 1.233 to 2.617). Using these factors, a high risk group of 26 patients was defined, with 19.2\% 1 year survival. Denying dialysis to this group would save $3.2 \%$ of the total cost of the chronic programme but would sacrifice five long term survivors. Less rigorous definition of the high risk group would save more money but lose more long term survivors. Conclusions Severity of comorbid conditions and functional capacity are more important than age in predicting survival and morbidity of patients on dialysis. Late referral for dialysis affects survival adversely. Denial of dialysis to patients in an extremely high risk group, defined by a new stratification based on logistic regression, would be of debatable benefit.

\section{Introduction}

Renal replacement therapy is expensive, and the number of patients receiving such treatment is predicted to rise by $50-100 \%$ over the next 15 years. ${ }^{1}$ Not surprisingly, the unrestricted availability of such treatment is being questioned. Three ways of reducing cost are to increase efficiency, to compromise on quality, or to reduce the numbers treated. ${ }^{2}$ It is generally accepted that implicit rationing is widely practised. ${ }^{3}$ Non-referral to nephrologists and non-acceptance by them both seem to occur. ${ }^{5-9}$ Wiltshire Health Authority was the first to publish criteria for the acceptance of patients on to their local renal replacement therapy programme. ${ }^{10}{ }^{11}$ Its aim was "to prioritise entry on to the programme for those patients who have the most likelihood of health gain from treatment, based on potential life years to be gained from treatment (anticipated as at least 12 months), absence of significant comorbidity, and the capability of independent living." They did not, however, define what comorbidity they considered to be significant or the point at which the capacity for independent living should be assessed.

Any guidelines to restrict the availability of treatment must be evidence based. We therefore performed a retrospective study of all patients entering our dialysis programme over a 4 year period, and studied survival and need for hospitalisation in relation to a number of factors. In particular we tried to define a high risk group, with a low likelihood of surviving 1 year, to allow us to question whether, as suggested by Hirsch and colleagues, dialysis might reasonably be withheld. ${ }^{12}$

\section{Subjects and methods}

\section{Catchment population}

The Lister renal unit, and its sister unit at St Albans, provides renal services for most of Hertfordshire and Bedfordshire and has a catchment population of about 1.15 million people. Peritoneal dialysis (both continuous ambulatory and automated) and haemodialysis are prescribed according to urea kinetic modelling. ${ }^{13}$ Patients on haemodialysis receive high flux treatment. Postdilutional haemodiafiltration is standard for patients with a residual urea clearance of $<1 \mathrm{ml} / \mathrm{min}$.

\author{
Department of \\ Nephrology, Lister \\ Hospital, Stevenage \\ SG1 $4 \mathrm{AB}$ \\ Shahid M Chandna, \\ associate specialist \\ Christopher \\ Lawrence, \\ medical student \\ Roger N \\ Greenwood, \\ consultant \\ Ken Farrington, \\ consultant \\ Department of \\ Psychology, \\ University of \\ Hertfordshire, \\ Hatfield AL10 9AB \\ Joerg Schulz, \\ senior lecturer \\ Correspondence to: \\ Dr Chandna \\ shahid.chandna@ \\ lister.org.uk
}

BMJ 1999;318:217-23 


\section{Patients studied}

Every patient with chronic renal failure who started dialysis between 1 April 1992 and 31 March 1996 and received even a single session of dialysis was included in the study. The only exception was patients who started dialysis elsewhere or who were transferred out to other units. We did not use formal criteria for acceptance on to the dialysis programme. Patients were assessed individually for their likelihood of benefit. Patients with chronic renal failure included a group with planned entry to the programme--that is, patients previously seen at least once in our clinic-and a group with unplanned entry to dialysis. The group with unplanned entry to dialysis consisted of patients presenting to us for the first time with acute uraemia who had either remained dependent on dialysis beyond 3 months or who had died during the first 3 months and whose renal failure was retrospectively deemed to be chronic. This judgment was made on the basis of the patient's history, ultrasonograms, and, where applicable, renal biopsy and post mortem findings. Data were examined in July 1997 to give a minimum follow up of 15 months.

\section{Method of study}

The records of all patients receiving renal replacement therapy in our hospital are entered on to a database (Di-Proton, Clinical Computing, London). We searched the database and case notes to obtain variables including:

- Age

- Sex

- Mode of initiation of dialysis (planned or unplanned)

- Date of starting dialysis

- Functional status according to the Karnofsky performance score,${ }^{14}$ both at the time of starting dialysis and 3 months before

- Number and severity of comorbid conditions. Each comorbid condition (cardiac disease, peripheral vascular disease, cerebrovascular disease, and respiratory disease) was graded according to symptom score. Cardiac disease was graded according to the New York Heart Association's functional classification. Other diseases were graded in a similar way: 1 indicating mild disease and 4 severe, disabling disease. Cirrhosis was graded as 4. Cancer was graded from 1 to 4 according to a matrix defined by its nature (medium term survival) and by its current state of activity. Scores of all comorbid conditions were combined to obtain a comorbidity severity score. The impact of diabetes was studied separately

- Number and duration of hospital admissions

- Date of death. ${ }^{78}$

Risk groups

Wright, and Khan and colleagues recommended stratification of patients into three risk categories to facilitate comparison of survival between units. ${ }^{15}{ }^{16}$ We used their scheme to compare results and to assess whether this stratification may be helpful in deciding when not to offer dialysis. We contrasted this approach with a newly developed system on the basis of logistic regression.

Cost

We calculated the cost of dialysis and hospitalisation to the nearest day: $£ 16614$ per patient per year for

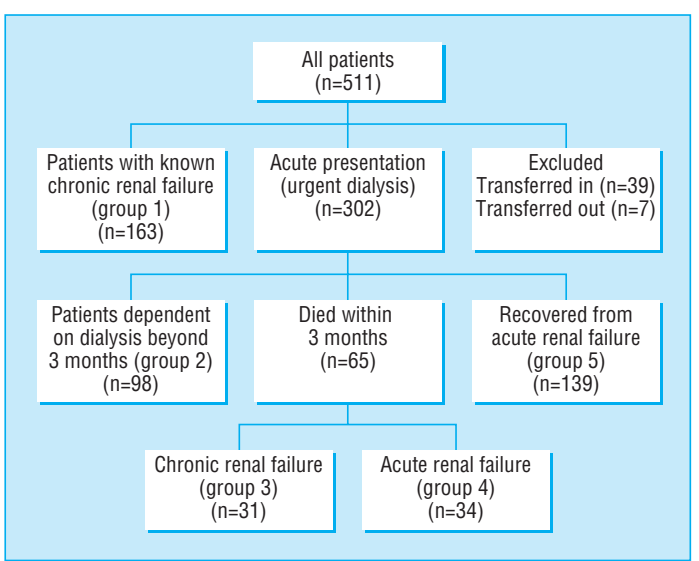

Fig 1 Study population comprising 292 patients from groups 1, 2, and 3

outpatients’ dialysis and $£ 250$ per day for inpatients’ treatment.

\section{Statistical methods}

We used the Kaplan-Meier method to compare the survival time between groups, and we assessed differences by the log rank test. We constructed Cox's proportional hazard models, after guidelines by Andersen, to estimate the impact of several risk factors, and to control for confounders. ${ }^{17}$ We performed logistic regression analysis to build a multivariable prediction equation for 1 year survival. We used spss release 8.0.0 for all statistical tests.

\section{Results}

\section{Patients studied}

During the 4 years of the recruitment period, 511 patients started dialysis. Of these, $39(8 \%)$ were transferred to us on dialysis from other units and seven $(1 \%)$ were transferred to other units during the study and could not be included. Figure 1 provides a breakdown of the remaining 465 (91\%) patients. Patients in groups 1 and 2 by definition had chronic renal failure. We excluded patients in group 5 because they had acute renal failure and did not require dialysis beyond 3 months. Of the 65 patients presenting acutely who died within 3 months, 31 (group 3) were thought to have chronic renal failure (16 had severe impairment of renal function before presentation, four had bilateral small kidneys, seven had myeloma, and four had long term dialysis access created or planned due to non-recovery of renal function). We excluded the remaining 34 (group 4), who were thought to have acute renal failure. The distinction between groups 3 and 4 was necessarily subjective because the data were retrospective. A concordance rate of $94 \%$ was, however, obtained on a repeat analysis by a second observer. We thus included all patients with chronic renal failure who were non-transferred $(n=292)$ and who received even a single session of dialysis. Entry into the dialysis programme was planned in $163(56 \%)$ patients and unplanned in $129(44 \%)$.

\section{Patient characteristics}

Data were available on all patients, and no substitution was needed. The mean age of the patients was 61.3 
years (18-92 years, SD 15.8). One hundred and ninety three $(66 \%)$ of the patients were male, $59(20 \%)$ had diabetes, $264(90 \%)$ were white, $17(6 \%)$ were Asian, and $11(4 \%)$ were Afro-Caribbean. Causes of renal failure included glomerulonephritis in $20 \%$ of patients, diabetic nephropathy in $15 \%$, polycystic kidneys in $10 \%$, chronic interstitial nephritis in 7\%, myeloma in $5 \%$, and others (including bilateral small kidneys) in 33\%.

\section{Comorbidity}

Cardiac disease was present in 110 patients (38\%), cerebrovascular disease in 34 (12\%), peripheral vascular disease in $70(24 \%)$, respiratory disease in $44(15 \%)$, liver cirrhosis in $3(1 \%)$, and malignancy (present or past) in $40(14 \%)$. Table 1 shows the comorbidity severity scores. The mean score was 2.1 (0-8, SD 2.2).

\section{Karnofsky performance score}

The mean Karnofsky performance score at the time of starting dialysis was 62.2 (10-90, SD 26.7): 96 patients $(33 \%)$ had scores $\leqslant 40$ (dependent, or requiring institutional or hospital care), 62 (21\%) had scores of 50-70 (requiring assistance), and $134(46 \%)$ had scores $\geqslant 80$ (able to carry on normal activity). The mean Karnofsky performance score 3 months before presentation was 86.6 (40-100, SD 15.1).

\section{Fate of patients}

During the 63 months follow up, 45 (15\%) patients received renal transplants (42 were functioning at last follow up) and eight (3\%) regained sufficient renal function after 3 months or more on dialysis to become independent of dialysis. Both groups were included in the survival analysis and were not censored at the end of dialysis requirement. One hundred and twenty nine (44\%) patients died: 39 (13\%) within 3 months of starting dialysis and $70(24 \%)$ within 1 year of starting dialysis. If only patients surviving beyond 3 months are included in the survival analysis, as is the case with other registries like USRDS (United States Renal Data Systems), the mortality rate in the first year of dialysis was $15 \%$.

\section{Group comparisons of survival}

Figure 2 shows the Kaplan-Meier survival curves for the four main determinants of survival. The survival curves of the four age groups differed significantly $(\mathrm{P}<0.001)$ except for the two highest age groups, which did not differ from each other (fig 2(a)). Median survival in the $>75$ age group was 19 months (95\% confidence interval 7.7 to 30.5 ), in the $65-75$ age group it was 29.4 months (19.4 to 39.4), and in the 50-65 age group it was 52 months (30.6 to 73.4). Median survival in the $<50$ age group is not available $(90 \%$ alive at last follow up).

Figure 2(b) shows the influence of comorbidity on survival. There was a significant difference in survival $(\mathrm{P}<0.0001)$ between patients with no comorbidity (median survival not available, $82 \%$ alive at last follow up) and patients with mild to moderate comorbidity

\section{Table 1}

Comorbidity severity score*

\begin{tabular}{|c|c|}
\hline Score & $\begin{array}{c}\text { No }(\%) \text { of } \\
\text { patients }\end{array}$ \\
\hline 0 & 107 (36.6) \\
\hline 1 & $21(7.2)$ \\
\hline 2 & $52(17.8)$ \\
\hline 3 & 39 (13.4) \\
\hline 4 & $29(9.9)$ \\
\hline 5 & $16(5.5)$ \\
\hline 6 & $15(5.1)$ \\
\hline 7 & $8(2.7)$ \\
\hline 8 & $5(1.7)$ \\
\hline
\end{tabular}

*Cardiac score, according to New York Heart Association, and respiratory disease score $(1-4)$, cerebrovascular disease score (1-4) peripheral vascular disease score (1-4) cirrhosis (4), and malignancy score (1-4)
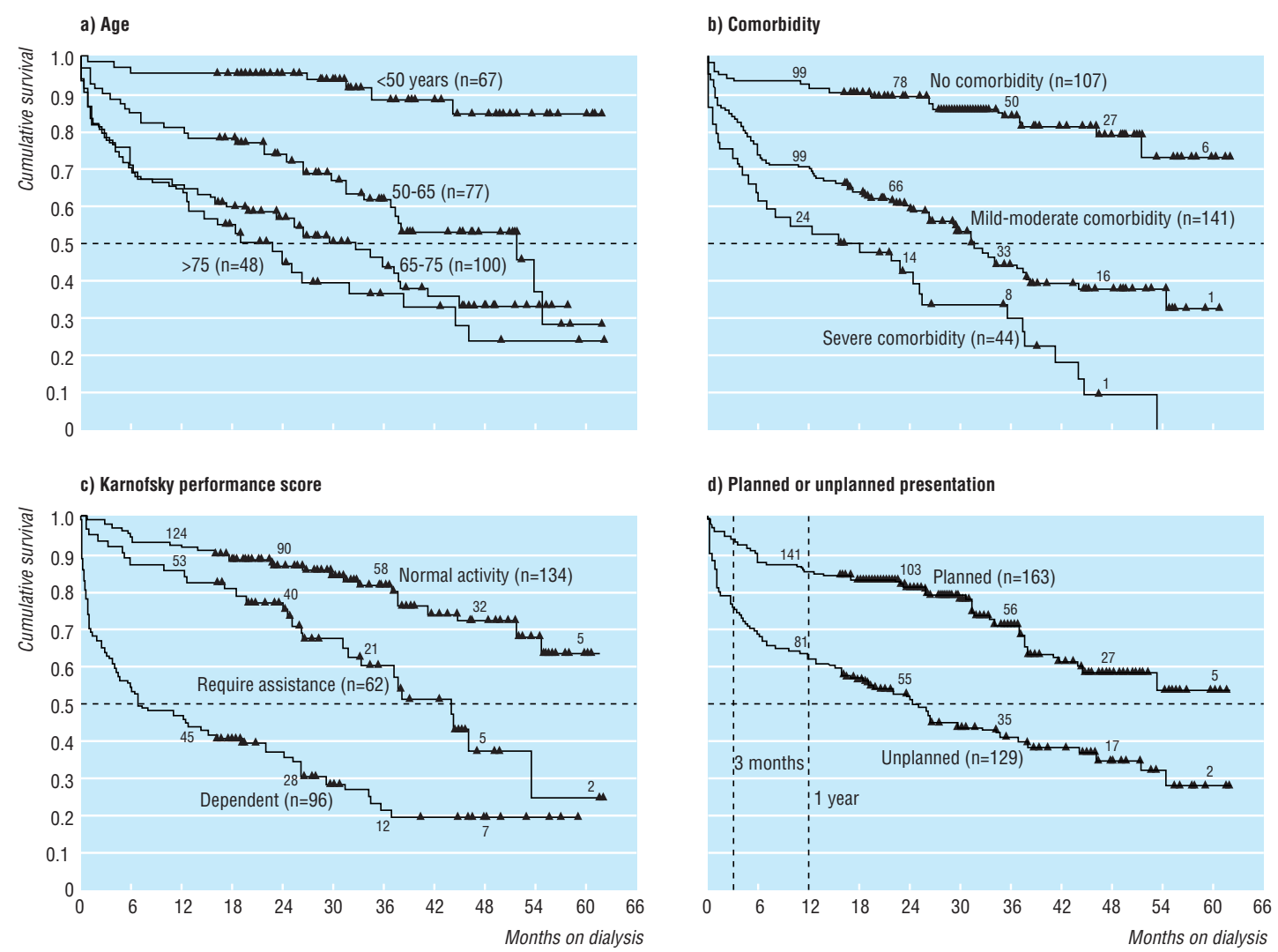

Fig 2 Kaplan-Meier survival curves. Each step represents one death, and each triangle denotes survivor at latest follow up. Numbers on top of lines are patients remaining in analysis at each time point. (a) Effect of age on survival. (b) Effect of comorbidity (using comorbidity severity score: mild-moderate comorbidity, 1-4; severe comorbidity, 5-8. (c) Survival in three groups defined by Karnofsky performance scale. (d) Difference in survival between planned and unplanned presentations for dialysis 


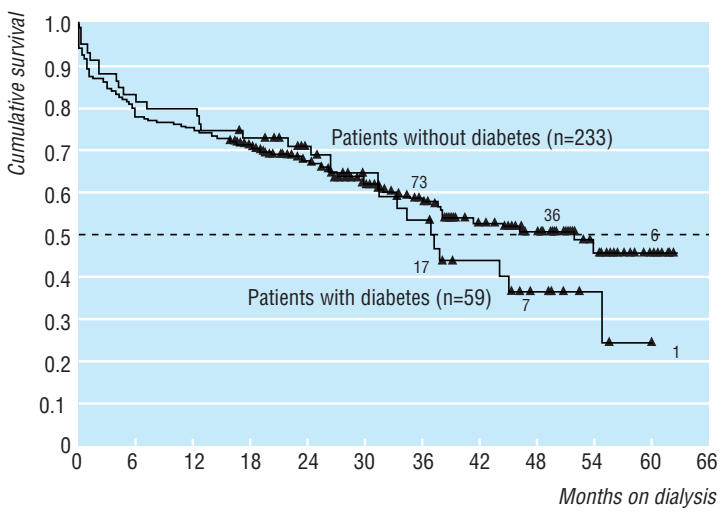

b)

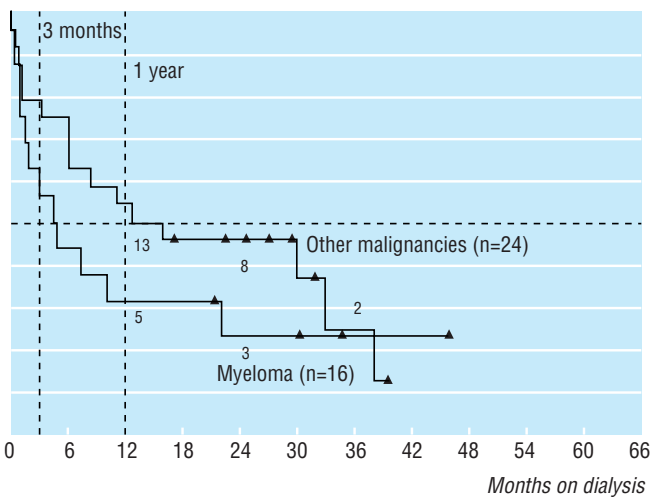

Fig 3 (a) Difference in survival between patients with and without diabetes. (b) Survival in patients with malignancies

(scores 1-4; median survival 31.6 months, 95\% confidence interval 25.0 to 38.1 ) or severe comorbidity (scores 5-8; 15.9, 0.0 to 32.6). The difference in survival was also significant $(\mathrm{P}=0.002)$ in patients with mild to moderate comorbidity or severe comorbidity. There was no significant difference in survival between patients with comorbid involvement of one system (median 31.5 months, 25.7 to 37.2) and with multiple system involvement $(24.7,12.3$ to 37.0$)$. The comorbidity severity score therefore discriminated better between comorbidity groups than the number of systems involved.

Figure 2(c) shows survival influenced by functional status at presentation. The median survival in the dependent group (Karnofsky performance score $10-40$ ) was 7.2 months (95\% confidence interval 0.7 to 13.7 ) and in the group requiring assistance (score 50-70) it was 44.3 months (36.3 to 52.2). Median survival in the normal activity group (score 80-100 at presentation) was not available ( $78 \%$ alive at last follow up). The difference among the three groups was statistically significant $(\mathrm{P}<0.001)$.

Figure 2(d) shows a difference in survival $(\mathrm{P}<0.0001)$ between patients whose dialysis was unplanned (median 24.7 months, 95\% confidence interval 18.6 to 30.8 ) and those in whom it was planned (median survival not available, 69\% alive at last follow up). The two survival curves did not become parallel till beyond 1 year.

Two specific comorbid conditions were of special interest. Figure 3(a) shows no significant difference in survival between patients with diabetes (median 37.2 months) and those without diabetes (median 52 months). Only $25 \%$ of patients with diabetes (versus $49 \%$ of patients without diabetes, $\mathrm{P}=0.001$ ) started dialysis in unplanned fashion. Patients with myeloma had a median survival of 4.5 months and a 1 year survival of $31 \%$. Five patients survived for more than 20 months (fig 3(b)). We could not find any factors that differentiated patients with a better prognosis.

\section{Multivariate analysis of survival}

The joint impact of all four main determinants (age, comorbidity severity score, Karnofsky performance score at presentation, and initiation of dialysis) on survival time was analysed using Cox regression. Sex was considered a possible moderator, and it was added to the list of predictors. The first model (M1, table 2) assessed all five predictors simultaneously but could be reduced to Mla without sex and unplanned entry to dialysis, since these predictors yielded no significant contribution, and model Mla was therefore chosen as the basis for further improvements. We then tested the effect of specific comorbidities in addition to the global comorbidity score. Three comorbid conditions (diabetes, cardiovascular diseases, and myeloma (this was chosen in preference to cancer, which consisted of a very diverse group dominated by myeloma)) were added to M1a resulting in model M2. However, only myeloma significantly affected survival whereas cardiac disease and diabetes could be removed from M2 without loss in predictive power (likelihood ratio $=1.2$; $\mathrm{df}=2, \mathrm{NS}$ ) leading to model M2a. Judged by the index $\mathrm{R}$ (to be read as a partial correlation), the most important factor in M2a was the Karnofsky performance score at presentation with a hazard ratio of 0.979 -that is, every 10 point decline in functional status is expected to increase the hazard of dying by $21 \%$. Similarly, each unit increase in the comorbidity score increased the risk of death by $24 \%$. Patients with myeloma had a 2.15-fold excess risk of dying.

To ascertain whether the functional capacity before the development of terminal uraemia is an indicator of the ability to benefit from renal replacement therapy, we looked at the Karnofsky performance score 3 months before dialysis, and the change in functional status in the subsequent 3 months. It was expected that further decline in functional status during the development of terminal uraemia would be negatively related to the ability to benefit from renal replacement therapy leading to shorter survival times. None of the patients had an improvement of functional status during this period: only 17 patients $(5.8 \%)$ maintained it whereas all the others deteriorated. In model M3 the Karnofsky performance score 3 months before was the most important prognostic factor with a hazard ratio of 0.962 - that is, death risk increased by $38 \%$ with every 10 points decrease in Karnofsky performance score. However, as expected, change in functional status was also significantly $(\mathrm{P}<0.001)$ related to survival. A 10 point decline in functional status in the 3 months before dialysis increased the likelihood of dying by $15 \%$ (hazard ratio 1.015). Compared with M2a, where only the Karnofsky performance score at presentation 
was taken into account, M3 is slightly more predictive (likelihood ratio $=8, \mathrm{df}=1, \mathrm{P}<0.001$ ) although the combined effect of the Karnofsky performance score at 3 months and change in functional status was very close to the estimate for the Karnofsky performance score at presentation in M2a. For a prognostic model of renal replacement therapy success, the extra information offered by M3 might therefore be dispensable. In a separate model, M4, we assessed the impact of unplanned presentation for dialysis, controlled for patients' age, the Karnofsky performance score 3 months before, and comorbidity score. Initiation of dialysis significantly contributed to the model (likelihood ratio $=9.5, \mathrm{df}=1, \mathrm{P}<0.01$ ) with unplanned presentation increasing the risk of dying by $80 \%$ (table 2 ).

Finally, we investigated whether there was any indication that the impact of the predictors in M2a was non-additive. Only the interaction between Karnofsky performance score at presentation and the comorbidity score could slightly improve the model (likelihood ratio $=4.6, \mathrm{df}=1, \mathrm{P}<0.03$ ), while all other interaction terms did not pass the entrance condition of the forward algorithm (i.e. $\mathrm{P}<0.05$ for a term to be included). Since the amount of non-additivity was very small it provided no substantial gain in clinical utility compared with M2a. Residual analysis showed some departure from the proportionality assumption for the factor myeloma but not for the covariates. The linearity assumption was not violated and there were no outliers.

\section{Predictors of 1 year survival}

We tried to develop a stratification to demarcate three groups of patients with different probabilities of 1 year survival, and we compared our scoring system with the three risk categories suggested by Wright, and Khan and colleagues. ${ }^{15}{ }^{16}$ We performed a logistic regression with respect to 1 year survival (yes/no) to generate a multivariable prediction equation. According to the results of the multivariate survival analysis, we chose age, comorbidity score, and functional status as the three most important predictors at presentation. The logistic regression model with these three predictors was highly significant (model $\chi^{2}=94.55, \mathrm{df}=3, \mathrm{P}<0.001$ ) and explained $28 \%$ of the log likelihood (we used Cox and Snell pseudo R square index). All three predictors were significantly related to 1 year survival: age (each year) (odds ratio $0.96,95 \%$ confidence interval 0.93 to 0.99 ; $\mathrm{P}<0.02)$, comorbidity score (each point) $(0.77,0.66$ to $0.89 ; \mathrm{P}<0.001$ ), and functional status (every 10 points on the Karnofsky performance score) (1.52, 1.32 to 1.74 ; $\mathrm{P}<0.001)$. In the next step, we partitioned the predicted probabilities of this model into three strata. We tried to obtain a high risk group with minimal chances of surviving 1 year, yet which was sufficient in size to generate potential cost savings if dialysis was not to be offered. Similarly, a low risk group was defined as having a maximum benefit from dialysis in terms of survival. The best cut off points for the predicted probabilities that fitted these criteria were $<0.36$ (high risk group of 26 patients) and $>0.94$ (low risk group of 98 patients) with a medium risk group $(n=168)$ in between. This stratification was significantly related to 1 year survival (likelihood ratio $=84.0 \mathrm{df}=2, \mathrm{P}<0.001$ ) and explained $25 \%$ of the $\log$ likelihood and had a proportional reduction in prediction errors of 23\%. By contrast, Wright, and Khan and colleagues' risk categorisation was also significantly related to 1 year survival (likelihood

Table 2 Cox regression models

\begin{tabular}{|c|c|c|c|c|c|c|c|c|}
\hline \multirow[b]{2}{*}{ Model specification } & \multicolumn{3}{|c|}{ Model fit* } & \multirow[b]{2}{*}{ Factors } & \multirow[b]{2}{*}{ Hazard ratio† $(95 \% \mathrm{Cl})$} & \multirow{2}{*}{$\begin{array}{c}\text { Wald } \\
\text { statistics }\end{array}$} & \multirow[b]{2}{*}{$P$ value } & \multirow[b]{2}{*}{ Index R‡ } \\
\hline & $\chi^{2}$ & df & $P$ value & & & & & \\
\hline \multirow[t]{5}{*}{ M1: Joint effect of all factors } & 139.6 & 5 & $<0.001$ & Age & 1.034 (1.016 to 1.052$)$ & 14.06 & $<0.001$ & 0.09 \\
\hline & & & & Comorbidity severity score & 1.238 (1.145 to 1.338$)$ & 28.96 & $<0.001$ & 0.14 \\
\hline & & & & KPS at presentation & 0.978 (0.969 to 0.987$)$ & 20.86 & $<0.001$ & -0.12 \\
\hline & & & & Male & 0.920 (0.630 to 1.343$)$ & 0.19 & 0.666 & 0.00 \\
\hline & & & & Unplanned presentation & 0.992 (0.619 to 1.591) & 0.00 & 0.973 & 0.00 \\
\hline \multirow[t]{2}{*}{ M1a: Reduced from M1 } & 137.4 & 3 & $<0.001$ & Age & 1.034 (1.016 to 1.051$)$ & 14.63 & $<0.001$ & 0.10 \\
\hline & & & & KPS at presentation & 0.978 (0.970 to 0.980$)$ & 37.63 & $<0.001$ & -0.16 \\
\hline \multirow{6}{*}{$\begin{array}{l}\text { M2: Specific effects by type of } \\
\text { comorbidity }\end{array}$} & 142.8 & 6 & $<0.001$ & Age & 1.037 (1.018 to 1.056) & 15.68 & $<0.001$ & 0.10 \\
\hline & & & & Comorbidity severity score & 1.241 (1.120 to 1.376$)$ & 16.96 & $<0.001$ & 0.11 \\
\hline & & & & KPS at presentation & 0.978 (0.971 to 0.986$)$ & 34.45 & $<0.001$ & -0.16 \\
\hline & & & & Myeloma & 2.187 (1.140 to 4.042$)$ & 4.91 & 0.027 & 0.05 \\
\hline & & & & Cardiac disease & $1.021(0.623$ to 1.675$)$ & 0.01 & 0.932 & 0.00 \\
\hline & & & & Diabetes & 1.130 (0.738 to 1.730$)$ & 0.32 & 0.573 & 0.00 \\
\hline \multirow{3}{*}{ M2a: Reduced from M2 } & & & & Comorbidity severity score & 1.240 (1.131 to 1.340$)$ & 29.70 & $<0.001$ & 0.15 \\
\hline & & & & KPS at presentation & 0.979 (0.972 to 0.986$)$ & 34.51 & $<0.001$ & -0.16 \\
\hline & & & & Myeloma & 2.150 (1.140 to 4.042$)$ & 5.59 & 0.030 & 0.05 \\
\hline \multirow{5}{*}{$\begin{array}{l}\text { M3: KPS } 3 \text { months before presentation, } \\
\text { and change in functional status }\end{array}$} & 149.6 & 5 & $<0.001$ & Age & 1.037 (1.020 to 1.055) & 17.64 & $<0.001$ & 0.11 \\
\hline & & & & Comorbidity severity score & 1.200 (1.114 to 1.304$)$ & 21.47 & $<0.001$ & 0.13 \\
\hline & & & & Myeloma & 3.186 (1.631 to 6.222) & 11.51 & $<0.001$ & 0.09 \\
\hline & & & & KPS 3 months before & $0.962(0.950$ to 0.974$)$ & 39.04 & $<0.001$ & -0.18 \\
\hline & & & & Change in KPS (3 months to present) & 1.015 (1.006 to 1.024$)$ & 11.57 & $<0.001$ & 0.09 \\
\hline \multirow[t]{4}{*}{ M4: Importance of early referral } & 141.3 & 4 & $<0.001$ & Age & $1.033(1.016$ to 1.050$)$ & 14.58 & $<0.001$ & 0.10 \\
\hline & & & & Comorbidity severity score & $1.232(1.142$ to 1.328$)$ & 29.30 & $<0.001$ & 0.15 \\
\hline & & & & KPS 3 months before & 0.970 (0.956 to 0.981) & 26.12 & $<0.001$ & -0.14 \\
\hline & & & & Unplanned presentation & 1.796 (1.233 to 1.617$)$ & 9.30 & 0.010 & 0.08 \\
\hline
\end{tabular}

KPS=Karnofsky performance score. *Global $\chi^{2}$ score to model. †Related to 1 year age increment, 1 point on the 8 point comorbidity severity score, and 1 point on the 100 point KPS. $\ddagger$ To be read like partial correlation. 


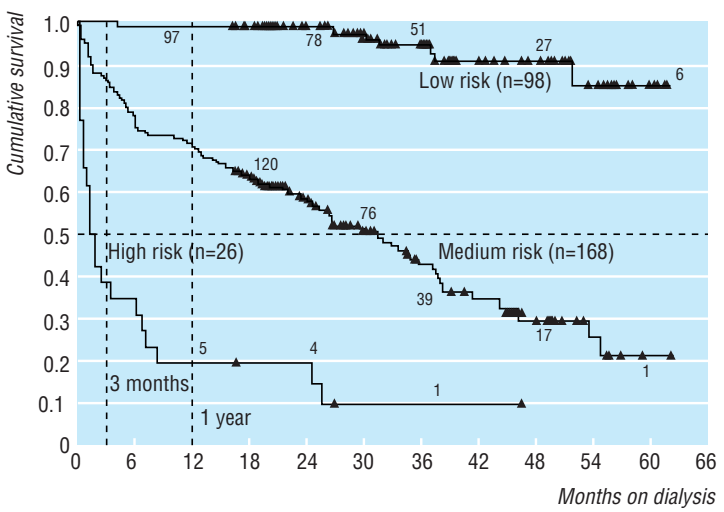

b)

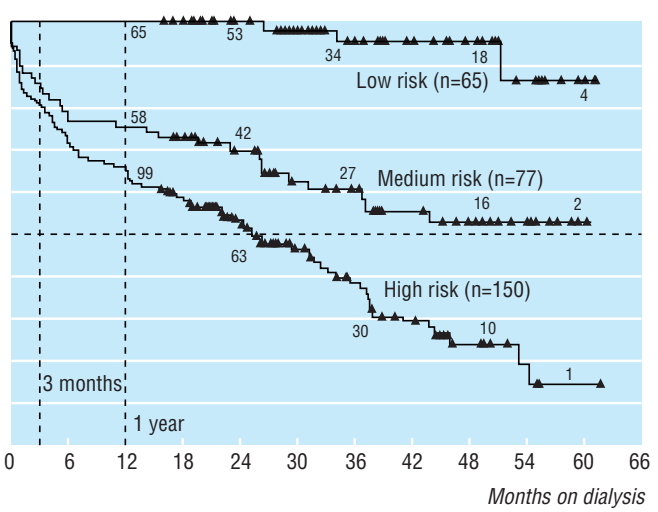

Fig 4 Risk stratification. Risk groups based on (a) logistic regression and (b) scheme by Wright, and Khan et $\mathrm{a}^{15} 16$

ratio $=43.3 \mathrm{df}=2, \mathrm{P}<0.001)$ but explained only $14 \%$ of the log likelihood $(\lambda=0 \%) .{ }^{15} 16$ The concordance between the two stratifications was moderate (Kendall's $\left.\tau_{c}=0.60\right)$. Furthermore, our stratification produced more pronounced differences in survival curves (fig 4). Using Khan and colleagues' system, only 65 of our patients $(22.3 \%)$ were in a low risk group (age $<70$, no comorbidity), 77 (26.4\%) were in a medium risk group, and $150(51.4 \%)$ were in a high risk group (age $>80$ or any age with two comorbid conditions or malignancy). Two year survival (for patients with at least 2 years potential follow up) was $100 \%, 66.7 \%$, and $55.7 \%$ respectively. Khan and colleagues' results were $86 \%$, $60 \%$, and $35 \%$ respectively. ${ }^{16}$

\section{Cost}

The cost of dialysis and hospitalisation of 26 high risk patients was 3.2\% of the total cost for all 292 patients throughout the time of the study. The bulk of this cost was that of dialysing the five long term survivors. If this was excluded, the actual "wasted cost" was $£ 128545$ (£32 136 per year) and was $0.99 \%$ of the overall cost. Raising the cut off point progressively to include all the medium risk patients resulted in greater savings at the cost of losing more potential survivors (table 3).

\section{Hospitalisation rate}

As a measure of morbidity on dialysis, we looked at the proportion of time spent in hospital after the initiation of dialysis. The time spent in hospital was inversely related to the logistic regression probability score $(r=-0.504, \mathrm{P}<0.0005)$. The 26 patients in the high risk group spent an average of $43.7 \%$ of their life in

Table 3 Cost reduction by exclusion of high risk patients

\begin{tabular}{llccc}
$\begin{array}{l}\text { Cut off } \\
\text { point* }\end{array}$ & Risk group & $\begin{array}{c}\text { No of patients } \\
\text { excluded } \\
\text { (\% of all patients) }\end{array}$ & $\begin{array}{c}\text { No of 1 year } \\
\text { survivors lost } \\
\text { (\% of excluded patients) }\end{array}$ & $\begin{array}{c}\text { Cost saving in } \mathfrak{z} \\
\text { (\% of total cost } \\
\text { over 5 years) }\end{array}$ \\
\hline 0.36 & High & $26(8.9)$ & $5(19.2)$ & $407495(3.2)$ \\
\hline 0.5 & Medium & $52(17.8)$ & $18(34.6)$ & $1227831(9.3)$ \\
\hline 0.7 & Medium & $100(34.3)$ & $49(49.0)$ & $3012482(23.3)$ \\
\hline 0.85 & Medium & $146(50.0)$ & $84(57.5)$ & $5182950(40.1)$ \\
\hline 0.94 & Medium & $194(66.4)$ & $125(64.4)$ & $7428667(57.4)$ \\
\hline
\end{tabular}

${ }^{*}$ Cut off points obtained from logistic regression equation.

Predictive probability $=\frac{\mathrm{e}^{\mathrm{z}}}{1+\mathrm{e}^{\mathrm{z}}}$

where $z=6.3565$ [constant] $-($ age $\times 0.0388)-($ comorbidity severity score $\times 0.2650)-([100-$ Karnofsky performance score $] \times 0.0418$ ) hospital (7.0\% in 1 year survivors). The figures for medium risk and low risk groups were $15.7 \%(5.5 \%)$ and $2.2 \%(1.7 \%)$ respectively.

\section{Discussion}

We have shown that age, comorbidity, and functional capacity are predictors of survival and morbidity of patients on dialysis. Age is often taken as the main factor in deciding whether or not to offer dialysis, but our study shows that it is heavily outweighed by the severity of comorbid conditions in predicting survival (one step on the eight point comorbidity severity score had a greater higher hazard ratio than an age increment of 6.5 years in the Cox's model). The number of comorbid systems did not discriminate as well as the comorbidity severity score. Presumably having one condition severely predicts a poorer prognosis than having two conditions of minimal or moderate severity. Functional status (defined by the Karnofsky performance score) at the time of presentation, 3 months before presentation, and the change in between were all useful in predicting survival. This suggests that the frequently asked question "How was the patient a few weeks ago before he or she became very unwell?" may be useful in deciding whether or not to offer dialysis.

This study suggests that the Wiltshire Health Authority recommendations may be a reasonable starting point in identifying potential factors upon which to base prioritisation decisions. Their recommendations are, however, couched in very general terms. To be of value in patient selection the terms require more definition. In this study we have attempted to isolate quantifiable factors around which such definitions can be based.

We found that diabetes was not a significant predictor of medium term survival. While a difference may appear with larger numbers of patients or longer follow up time, the lack of difference can also be explained by the beneficial effect of early referral in patients with diabetes. Patients with diabetes often commence dialysis with lower serum creatinine concentrations than those patients without diabetes, although whether this reflects better residual function is unclear. ${ }^{18}$ Myeloma confers a very poor survival, but $31 \%$ of patients survived for more than 22 months (figure 3(b)) supporting our and others decisions to offer 
dialysis to these patients. ${ }^{19}$ Survival seems to be better in patients with other malignancies.

Like others, we have shown that late referral for dialysis is a major determinant of poor survival. ${ }^{20}{ }^{21} \mathrm{It}$ is rather depressing that, 13 years after Ratcliffe's paper, $44 \%$ of referrals still occur with patients in the terminal stages of uraemia. ${ }^{21}$ In a moribund patient it is difficult to decide whether treatable uraemia or untreatable comorbidity predominates. It may be that some patients are dialysed who may have been treated less aggressively had there been an opportunity to review them in the preterminal state. Apart from this, the severity of uraemia at presentation may itself affect survival. ${ }^{18}$ Late referral is also responsible for a significant workload and expense. ${ }^{22}{ }^{23}$ We agree with the NHS consensus statement that recommends nephrology referral at a serum creatinine concentration $>1.5 \mathrm{mg} /$ $\mathrm{dl}(135 \mu \mathrm{mol} / \mathrm{l})$ in women and $2.0 \mathrm{mg} / \mathrm{dl}(180 \mu \mathrm{mol} / \mathrm{l})$ in men. ${ }^{24}$

We realise the limitations of the retrospective nature of the study. Judging the potential reversibility of renal failure is not easy in patients who died during attempted resuscitation. Similarly, despite every effort, one can not guarantee the accuracy of retrospective comorbidity and Karnofsky performance score data. Like others, however, we believe that retrospective categorisation serves a useful purpose at least as an incentive for prospective work. ${ }^{165}$ While it is possible that the definition of high risk patients can be improved by prospective study, the method of risk stratification by a quantifiable decision model described here seems to be better in predicting survival and the need for hospitalisation than methods described previously. ${ }^{15} 16$

We have shown that while not practising formal rationing, we have been fairly rational in our decisions to offer dialysis. This is evidenced by good survival in low and medium risk patients who comprise most patients entering the programme. We have been able to question whether overt exclusion of a small number of high risk patients, identifiable at presentation by use of a new stratification encompassing functional capacity, comorbidity, and age, and likely to have a high level of hospitalisation and poor survival, would be justified on the basis of outcome and economics. Our data suggest that exclusion of even this high risk group would sacrifice a significant number of long term survivors, with negligible financial benefit. Moving the cut off point for high risk upward to save more money would lose many more lives (table 3). It is possible that an ultra high risk group was not even referred, and if they were, like Hirsch and colleagues, we did not offer them dialysis. $^{12}$

There is a pressing need for prospective studies in this field and to test our classification approach by logistic regression in other patient populations and settings. We suggest that, in the meantime, continued efforts to promote early referral and to reduce the unit cost of dialysis are likely to produce more financial savings than attempts to overtly ration access to dialysis, at least in the short term.

Contributors: SMC contributed to data validation and analysis, writing the paper, and preparation of graphs and tables. JS gave advice on statistical models, performed the multivariate data analysis, wrote up the results, and helped revise the paper. CL collected the data, performed the initial analysis of the data and presented the results to an internal meeting, wrote the first
- Functional status (assessed by Karnofsky performance score), severity of comorbid conditions, and age affect survival on dialysis

- Late referral is an important factor in poor survival and high costs

- Rationing of dialysis on the basis of age alone is unjustified

- A high risk group can be defined by logistic regression analysis using functional status, severity of comorbid conditions, and age

- Limited cost savings can be generated by denying access to dialysis to this high risk group, but long term survivors are sacrificed

draft of the paper with SMC, and reviewed the final manuscript RNG contributed to the inception of the study and cost analysis and reviewed the paper. KF contributed to the inception of the study, study design, and data validation and analysis, and reviewed the paper and rewrote sections. SMC, RNG, and KF will act as guarantors for the paper.

Funding: None.

Competing interests: None.

1 Davies R, Roderick P. Predicting the future demand for renal replacement therapy in England using simulation modelling. Nephrol Dial Transplant 1997;12:2512-6.

2 Hull AR. The legislative and regulatory process in the end-stage renal disease (ESRD) program, 1973 through 1997. Semin Nephrol 1997;17:160-9.

3 Kjellstrand CM, Moody H. Hemodialysis in Canada: a first-class medical crisis. Can Med Assoc J 1994;150:1067-71.

4 Friedman EA. Rationing of uremia therapy. Artificial Organs 1992;16:90-7.

5 Mendelssohn DC, Kua BT, Singer PA. Referral for dialysis in Ontario Arch Intern Med 1995; 155:2473-8.

6 Feest TG, Mistry CD, Grimes DS, Mallick NP. Incidence of advanced chronic renal failure and the need for end stage renal replacement treatment. BMJ 1990;301:897-900.

7 McGeown MG. Prevalence of advanced renal failure in Northern Ireland. BMJ 1990;301:900-3.

8 Parry RG, Crowe A, Stevens JM, Mason JC, Roderick P. Referral of elderly patients with severe renal failure: questionnaire survey of physicians. $B M$ J 1996;313:466.

9 Kilner JF. Selecting patients when resources are limited: a study of US medical directors of kidney dialysis and transplantation facilities. Am J Public Health 1988;78:144-7.

10 Farmery E, Milner P. Renal replacement therapy:purchasing review and recommendations. Devizes: Wiltshire Health Authority, 1997.

11 Brindle D. Budget special: health: straits talking. Guardian 2 July 1997;G2:6.

12 Hirsch DJ, West ML, Cohen AD, Jindal KK. Experience with not offering dialysis to patients with a poor prognosis. Am J Kid Dis 1994;23:463-6.

13 Sargent JA, Gotch FA. Principles and biophysics of dialysis. In: Maher JF, ed. Replacement of renal function by dialysis. Dordrecht: Kluwer, 1989:87-143.

14 Karnofsky DA, Abelman WH, Craver LF, Burchenal JH. The use of nitrogen mustards in palliative treatment of carcinoma. Cancer 1948;1:634-56. 15 Wright LF. Survival in patients with end-stage renal disease. Am J Kidne Dis 1991;17:25-8.

16 Khan IH, Catto GRD, Edward N, Fleming LW, Henderson IS, Macleod AM. Influence of coexisting disease on survival on renal-replacement therapy. Lancet 1993;341:415-8.

17 Andersen PK. Survival analysis 1982-1991: the second decade of the proportional hazards regression model. Stat Med 1991;10:1931-41.

18 Tattersall J, Greenwood R, Farrington K. Urea kinetics and when to commence dialysis. Am J Nephrol 1995;15:283-9.

19 Brown JH, Maxwell AP, Bruce I, Murphy BG, Doherty CC. Renal replacement therapy in multiple myeloma and systemic amyloidosis. Ir J Med Sci 1993;162:213-7.

20 Innes A, Rowe PA, Burden RP, Morgan, AG. Early deaths on renal replacement therapy: the need for early nephrological referral. Nephrol Dial Transplant 1992;7:467-71.

21 Ratcliffe PJ, Phillips RE, Oliver DO. Late referral for maintenance dialysis. BMJ 1984;288:441-3.

22 Jungers P, Zingraff J, Albouze G, Chauveau P, Page B, Hannedouche T, et al. Late referral to maintenance dialysis: detrimental consequences. Nephrol Dial Transplant 1993;8:1089-93.

23 Ifudu O, Dawood M, Homel P, Friedman EA. Excess morbidity in patients starting uraemia therapy without prior care by a nephrologist. Am J Kidney Dis 1996;28:841-5.

$24 \mathrm{NIH}$ consensus conference statement. Morbidity and mortality of renal dialysis. Ann Intern Med 1994;121:62-70.

25 Catalano C, Goodship THJ, Graham KA, Marino C, Brown AL, Tapson JS, et al. Withdrawal of renal replacement therapy in Newcastle upon Tyne: 1964-1993. Nephrol Dial Transplant 1996;11:133-9.

(Accepted 27 November 1998) 